\title{
Cuidar de Quem Cuida: Ferramentas de Avaliação dos Cuidadores
}

\section{Caring for Carers: Caregiver Assessment Tools}

Ana Carla Guimarães ${ }^{1}$, Lúcia Freitas ${ }^{1}$, Sílvia Orange Costa¹ Vera Brandão $^{1}$

\section{RESUMO}

O envelhecimento populacional é uma realidade, sendo que o aumento da esperança média de vida acarreta maior morbilidade nos indivíduos idosos e, consequentemente, um maior grau de dependência por parte destes. Na sequência destas alterações demográficas surgem alterações estruturais em termos familiares, com necessidade de estabelecer um prestador de cuidados para determinado indivíduo dependente.

As repercussões associadas ao processo de cuidar são várias, atingindo diversas áreas da vida do cuidador. Existem disponíveis vários instrumentos para avaliação da sobrecarga do cuidador: Carer Assessment of Difficulties Index, Carer Assessment of Satisfactions Index, Carer Assessment of Managing Index, Carers' Assessment of Managing Index, Escala de avaliação da Satisfação com o Suporte Social e a Burden Interview Scale de Zarit. Estas avaliam diferentes áreas da vida do cuidador, desde a saúde física, mental, área social e mesmo financeira. Estes instrumentos devem ser utilizados de forma sistemática para identificar situações de sobrecarga precocemente.

PALAVRAS-CHAVE: Avaliação de Necessidades; Cuidadores; Envelhecimento; Idoso 


\section{ABSTRACT}

As the world population is getting older, the average life expectancy increases, leading to greater morbidity in the elderly and, consequently, a greater dependence in their day-to-day lives.

Following these demographic changes comes structural family changes, including a need to provide a personal caregiver to these dependent individuals.

There are many repercussions associated with this process, affecting several areas of the caregiver's life.

There are several tools available for caregiver burden assessment: Carer Assessment of Difficulties Index, Carer Assessment of Satisfaction Index, Carer Assessment of Managing Index, Carers' Assessment of Managing Index, Social Support Satisfaction Rating Scale and Burden Interview Scale from Zarit. These instruments aim to assess the different areas of the caregiver's life, such as physical, mental, social and even financial health. They should be used systematically to identify overload situations in early stages.

KEYWORDS: Aged; Aging; Caregivers Needs Assessment

O envelhecimento da sociedade é uma realidade incontornável, à qual se associam, em função da maior longevidade, maior morbilidade da população e, consequentemente, maiores índices de dependência.

De acordo com a Organização Mundial de Saúde (OMS), em 2020 prevê-se que tenhamos em todo o mundo um bilião de pessoas idosas, sendo a maioria nos países em desenvolvimento. ${ }^{1}$ Segundo dados do Instituto Nacional de Estatística, em Portugal, os números relativos ao índice de envelhecimento e de dependência de idosos, tem seguido o perfil crescente que se verifica na União Europeia, atingindo em 2018 os valores de 157,4 e 33,6, respetivamente. $^{2}$

O aumento da esperança média de vida acarreta mudanças na sociedade que atingem as estruturas familiares, os serviços de saúde e a segurança social.

Quando o envelhecimento está associado à dependência, é fundamental criar as condições necessárias para que a assistência nas atividades de vida diária promova a dignidade do alvo dos cuidados.

A prestação de cuidados a um idoso dependente é multidimensional e pode assumir 2 formas distintas: cuidado formal (levada a cabo por profissionais da área) ou cuidado informal. Os cuidados informais são executados preferencialmente no domicílio, habitualmente levados a cabo por familiares, vizinhos, amigos ou outros que são denominados cuidadores informais. A literatura define 3 níveis de cuidadores informais 3 :

- Cuidador primário ou principal: É o elemento sobre o qual está depositada a responsabilidade pela prestação de cuidados, ou seja, tem a função de supervisionar, orientar, acompanhar e/ou zelar diretamente pela pessoa idosa que necessita de cuidados. Este é quem realiza a maioria dos cuidados.
- Cuidador secundário: É alguém que coopera na prestação de cuidados ocasionalmente, mas não tem a responsabilidade de cuidar. Habitualmente são familiares que dão apoio ao cuidador principal, em determinadas situações.

- Cuidador terciário: É um familiar, amigo ou vizinho que ajuda muito raramente ou apenas quando solicitado, geralmente em situações de emergência, mas não tem nenhuma responsabilidade pelo desenvolvimento do ato de cuidar do idoso dependente.

Tornar-se cuidador é sempre um processo idiossincrático que depende de muitos fatores. Segundo Martin, existem 4 fatores essenciais na escolha do cuidador informal: a relação familiar, a co-residência ou proximidade geográfica, o género do idoso dependente e potencial cuidador e condicionantes relativas a descendentes. ${ }^{4}$ Assim, a escolha do cuidador é um processo dinâmico, determinado por fatores sociais, laborais, emocionais e económicos, entre outros.

As repercussões associadas ao processo de cuidar são visíveis em diversos contextos da vida do cuidador. A sobrecarga do cuidador define-se pelo conjunto de consequências que ocorrem na vida do prestador de cuidados, na sequência de um contacto próximo com um dependente. As repercussões associadas ao cuidar conceptualizam-se em sobrecarga objetiva (alterações de parâmetros da vida do cuidador observáveis e quantificáveis - física, económica, etc.) e sobrecarga subjetiva (sentimentos e emoções de como cada um responde face à experiência). 3.5

A experiência de cuidar poderá comprometer a dinâmica familiar, pois o idoso dependente passa muitas vezes a ser o centro das atenções, o que implica alterações significativas, nomeadamente, no ambiente doméstico. 
O impacto dos cuidados dedicados a idosos dependentes pode ser no sistema familiar reconhecido como uma experiência emocional e fisicamente cansativa, podendo interferir na qualidade de vida dos cuidadores. Muitas vezes poderá implicar modificações na própria habitação e a perda temporária ou permanente de emprego pelo cuidador. A unidade familiar sofre uma rutura da rotina de vida, privacidade e dos rendimentos. Poderão surgir também, sentimentos de ansiedade, desespero, cansaço, e desamparo que são frequentes em famílias de idosos dependentes. A convivência com uma pessoa dependente, exige uma série de aprendizagens diárias e adaptações. Cuidar é uma atividade complexa com dimensões éticas, psicológicas, sociais, demográficas e que também tem os seus aspetos clínicos, técnicos e comunitários. ${ }^{6} \bigcirc$ cuidador informal enfrenta, diariamente, uma grande diversidade de tarefas, as quais são definidas pela doença e/ou incapacidade funcional do idoso. Muitas vezes, sem perceber, o cuidador sofre de sobrecarga e esquece que é uma pessoa que também necessita de cuidados.

Posto isto, os prestadores de cuidados deverão ser ajudados pelos profissionais de saúde, a lidar com os sentimentos de culpa, tristeza, frustração, raiva, depressão e outros que surjam dessa responsabilidade assumida. As avaliações do cuidador, sobre a situação de cuidar e seus efeitos sobre a saúde física e mental, podem ajudar a identificar a exposição do cuidador aos fatores de stress, a sua vulnerabilidade e os seus recursos psicológicos e sociais. Estes podem tornar-se progressivamente mais importantes devendo ser avaliados à medida que as necessidades do idoso dependente aumentam.

Na avaliação do cuidador informal devem ser considerados os diferentes contextos em que o cuidador pode encontrar dificuldades, ainda que não se aperceba delas. Uma avaliação sistematizada e recorrente das dificuldades vivenciadas, das estratégias adotadas, da sobrecarga sentida, do suporte social e familiar podem ajudar a identificar cuidadores em risco de doença.

O Carer Assessment of Difficulties Index (CADI), o Carer Assessment of Satisfactions Index (CASI) e o Carer Assessment of Managing Index (CAMI) são um conjunto de questionários criados no Reino Unido e traduzidos e validados para português em 2002. O objetivo da sua aplicação é avaliar as dificuldades percecionadas, as fontes de satisfação e as estratégias encontradas. ${ }^{7,8}$

O CADI desenvolvido por Mike Nolan, no Reino Unido, adaptado e validado para a população portuguesa por vários investigadores, permite avaliar as dificuldades sentidas pelos cuidadores. É constituído por 30 poten- ciais dificuldades relacionadas com o cuidar do idoso e suas implicações na vida social, na saúde, na situação económica, no relacionamento e no apoio profissional ao cuidador. Este instrumento permite a identificação do tipo de dificuldades nas situações de prestação de cuidados ao idoso, bem como a forma como cada prestador de cuidados perceciona e vivencia essas dificuldades. Segundo Sequeira (2010) o CADI compõe-se de sete fatores: problemas relacionais com o idoso, restrições na vida social, exigências de ordem física da prestação de cuidados, reações à prestação de cuidados, falta de apoio familiar, falta de apoio profissional e problemas financeiros. $^{7-9}$

Nolan e colaboradores (1996) utilizaram o seu estudo - Carers' Assessment of Satisfactions Index para avaliar a satisfação sentida pelos cuidadores informais. O CASI é composto por 30 itens relacionados com aspetos positivos associados à prestação de cuidados e que são identificados pelo cuidador como fonte de satisfação. As diferentes fontes de satisfação podem ser agrupadas em três categorias: fontes de satisfação relacionadas com a dinâmica interpessoal, que podem ter como alvo o cuidador, a pessoa cuidada ou ambos; fontes de satisfação relacionadas com a dinâmica intrapessoal que podem ter como alvo o cuidador, a pessoa cuidada ou ambos; fontes de satisfação relacionadas com a dinâmica de resultados que tem como alvo o cuidador e a pessoa cuidada. ${ }^{10}$

O Carers' Assessment of Managing Index é um índice de avaliação de como os prestadores de cuidados enfrentam as dificuldades. O CAMI inclui 38 afirmações relacionadas com a forma como cada prestador de cuidados resolve e/ou atenua as dificuldades percecionadas. 0 cuidador tem ainda a possibilidade de referir a sua perceção sobre o grau de eficácia em cada situação. Reporta-nos para o facto de os cuidadores utilizarem ou não determinadas estratégias e para o grau de eficácia das estratégias adotadas. Este instrumento tem como objetivos conhecer a forma como cada prestador de cuidados, lida com as dificuldades percecionadas, que mecanismos mobiliza para lidar com esses acontecimentos adversos e quão adequados e eficazes eles são. 7,8,11

A Escala de avaliação da Satisfação com o Suporte Social (ESSS) permite avaliar a satisfação do cuidador com o suporte recebido (da família, amigos e das atividades sociais). A Escala de Satisfação com o Suporte Social, desenvolvida e validada por Ribeiro (1999), consiste numa escala de autopreenchimento, composta por 15 frases que permitem ao sujeito assinalar o seu grau de concordância com cada uma delas. A ESSS permite extrair quatro dimensões: Satisfação com amigos/amizades; 
Intimidade; Satisfação com a família e Atividades Sociais. A cotação total da escala varia entre 15 e 75, e uma pontuação mais alta corresponde a uma perceção de maior suporte social. ${ }^{11}$

A avaliação da sobrecarga do cuidador é efetuada com recurso à Escala de Sobrecarga do Cuidador, traduzida e adaptada para a população portuguesa a partir da Burden Interview Scale de Zarit. ${ }^{3}$ É um instrumento fiável, que possui boas características psicométricas para avaliar a sobrecarga do indivíduo e que pode ser utilizado com vários objetivos: como instrumento de diagnóstico (avaliação do risco) ou como instrumento de avaliação e monitorização. É um instrumento que permite avaliar a sobrecarga objetiva e subjetiva do cuidador informal com informações sobre: saúde, vida social, vida pessoal, situação financeira, situação emocional e tipo de relacionamento. É constituído por 22 afirmações com 5 possibilidades de resposta: nunca, raramente, às vezes, bastantes vezes e quase sempre e permite, no final, obter um score de sobrecarga consoante o somatório das respostas às afirmações. ${ }^{6}$

Com intuito semelhante existe também o Questionário de Avaliação da Sobrecarga do Cuidador Informal (QAS$\mathrm{Cl}$ ), que inclui sete sub-itens: implicações na vida pessoal, sobrecarga financeira, reação a exigências, satisfação com o papel e o familiar, suporte familiar, perceção dos mecanismos de eficácia e controlo. ${ }^{11}$

A Medicina Geral e Familiar, pela sua abrangência familiar e comunitária, é a especialidade de excelência para avaliação dos cuidadores. Nos cuidados de saúde secundários as atenções estão sobretudo viradas para o idoso, suas comorbilidades e intercorrências. O Médico de Família deve olhar a dependência de um indivíduo como um todo e, ter atenção à saúde física, psicológica e mental dos cuidadores. Uma avaliação rotineira destes prestadores de cuidados, com recurso a uma ou mais das ferramentas apresentadas, permitirá identificar problemas dos cuidadores precocemente e intervir atempadamente.

CONFLITOS DE INTERESSE: Os autores declaram não ter qualquer conflito de interesse na realização do presente trabalho.

FONTES DE FINANCIAMENTO: Não houve qualquer fonte de financiamento na realização do presente trabalho.

PROVENIÊNCIA E REVISÃO POR PARES: Não comissionado; revisão externa por pares.

CONFLICTS OF INTEREST: The authors declare that they have no conflicts of interest.
FINANCIAL SUPPORT: This work has not received any contribution, grant or scholarship.

PROVENANCE AND PEER REVIEW: Not commissioned; externally peer reviewed.

\section{REFERÊNCIAS}

1. Resumo Executivo Envelhecimento no Século XXI: Celebração e Desafio; Fundo de População das Nações Unidas (UNFPA) e HelpAge International, 2012. [consultado Nov 2019] Disponível em: http://www.unfpa.org

2. Instituto Nacional Estatística. Projeções de População Residente - 2015-2080. Lisboa: INE; 2018.

3. Martins T, Peixoto M, Machado P, Araújo M. A Pessoa Dependente \& o Familiar Cuidador. Porto: Escola Superior de Enfermagem do Porto; 2006

4. Martín I. O cuidado informal no âmbito social. In: Paúl C, Fonseca A, editores. Envelhecer em Portugal. Lisboa: Climepsi; 2005.

5. Cardoso I. Perspectivas atuais sobre a sobrecarga do cuidador em saúde mental. Rev Esc Enferm. 2012; 46.

6. LAROZ E. Factores asociados al nível de sobrecarga de los cuidadores informales de adultos mayores dependientes. Redalyc. 2009; 18.

7. Sequeira C. Cuidar de Idosos com dependência física e mental. Lisboa: Lidel Edições Técnicas Lda; 2010.

8. Sampaio F, Sousa L, Neves P, Costa R, Pimenta S, Sequeira C. CASI, CADI e CAMI: Aferição das propriedades psicométricas das versões reduzidas na população portuguesa. Conference NursID Week. Semana de Investigação em Enfermagem. Escola Superior de Enfermagem do Porto 9 a 13 de julho 2018, Porto, Portugal.

9. Charlesworth GM, Tzimoula XM, Newman SP. Carers Assessment of Difficulties Index (CADI): psychometric properties for use with carers of people with dementia. Aging Ment Health. 2007;11:218-25.

10. Machado SC. Avaliação da sobrecarga do cuidador informal, no desempenho de suas funções, à pessoa idosa dependente, no concelho de Santana [tese de mestrado]. Madeira: Centro de Competências das Tecnologias da Saúde; 2012. [consultado Nov 2019] Disponível em: http://digituma.uma.pt/bitstream/10400.13/587/1/MestradoSorayaMachado.pdf

11. Artraso B, Goñi A, Biurrun A. Cuidados informales en la demencia: predicción de sobrecarga en cuidadoras familiares. Rev Esp Geriatria Gerontol Pamplona. 2003;38: 212-8. 